\title{
Combining Ability Analysis in Iron and Zinc Rich Rice Genotypes
}

\author{
N. Lingaiah", N. Sarla, V. Venkanna, Ch. Surender Raju, \\ K. Radhika and D. Vishnu Vardhan Reddy
}

Regional Agricultural Research Station, Warangal, Telangana State-506 007, India

*Corresponding author

\begin{abstract}
A B S T R A C T
Keywords

Iron and Zinc, Rice genotypes

Article Info

Accepted:

26 April 2018

Available Online:

10 May 2018

In the present investigation, the magnitude of specific combining ability variances was higher than that of general combining ability for all the characters under study except spikelet fertility (\%), kernel length, kernel length/breadth ratio and kernel elongation ratio indicating greater role of non- additive gene action in controlling most of the traits.
\end{abstract}

\section{Introduction}

The success of any breeding programme depends on selection of parents for hybridization and the choice of crosses to be advanced from the several crosses effected. This indicates that prior information on genetic background of the parents to be combined through crossing is very important. One of the techniques, widely used to extract information about the genetic system governing the inheritance of attributes, is the diallel analysis (Arunachalam, 1976). This design gives an idea about the relative magnitude of additive and non-additive components of heritable variance in expression of a trait. In case of diallel analysis developed by Griffing (1956) the additive and dominance components can be studied precisely in the absence of epistasis. Super crosses and parents with high $S C A$ effects and $G C A$ effects can be picked up.

\section{Materials and Methods}

The present investigation was carried out at Regional Agricultural Research Station, Warangal, which is located at an altitude of $304 \mathrm{M}$ above MSL, $17.97^{\circ} \mathrm{N}$ latitude and $79.60^{\circ} \mathrm{E}$ longitude in consecutive two seasons viz. Kharif, 2014, Rabi, 2014-15. Kharif (June - Dec) and Rabi (Nov - May) are the main crop seasons in this state can be called as rainy and post rainy seasons. The experimental material for heterosis and combining ability studies comprised of 10 genotypes viz., MTU 1010, WGL-32100, Ramappa, RP-Bio-5478270, RP-Bio-5478-166, RP-Bio-5478-176, 
DRR Dhan-40, RP-Bio-5478-185, SM- 686, SM-787, their $45 \mathrm{~F}_{1}$ hybrids generated from ten parents crossed (in diallel fashion without reciprocals during kharif, 2014) and two promising check varieties viz. BPT 5204 and Chittimuthyalu. The data obtained from $F_{1} s$ and parents were analysed as per Method II $\left(\mathrm{F}_{1} \mathrm{~S}+\right.$ parents) and Model - I (fixed effect) of Griffing (1956a) for combining ability. Analysis of variance for combining ability for yield, yield components (Table 1) indicated that general combining ability $(G C A)$ mean squares were significant for all the characters. For specific combining ability $(S C A)$, mean squares were significant for all the characters, except for spikelet fertility (\%) and kernel breadth. In the present investigation, the magnitude of specific combining ability variances was higher than that of general combining ability for all the characters under study except spikelet fertility (\%), kernel length, kernel length/breadth ratio and kernel elongation ratio indicating greater role of nonadditive gene action in controlling most of the traits.

The specific combining ability effects, estimates of $g c a$ and sca variances and their ratios of $45 \quad F_{1}$ hybrids for yield and its components are presented in Table 2.

\section{Results and Discussion}

Overall results indicated that the grain yield per plant and its components except spikelet fertility percentage were chiefly governed by non-additive gene action, as the $S C A$ variances were higher than the corresponding $G C A$ variances. For days to $50 \%$ flowering and plant height, the crosses with significant negative SCA effects are desirable. Accordingly, the crosses showing high SCA effects with high per se performance in desirable negative side viz., MTU 1010 x RPBio-5478-185 (high GCA x low GCA), WGL$32100 \times$ Ramappa (low $x$ high) for days to $50 \%$ flowering and the crosses, MTU $1010 \mathrm{x}$
Ramappa (high x low), RP-Bio-5478-270 x DRR Dhan-40 (low x high), RP-Bio-5478-176 x SM-787 (high x low), DRR Dhan-40 x RPBio-5478 -185 (high $x$ medium) and DRR Dhan-40 x SM-787 (high x low) for plant height were highly useful for evolving genotypes with shorter duration and short stature. These crosses showing high $\mathrm{x}$ low or vice versa indicated the involvement of mostly additive $\mathrm{x}$ dominant interactions could be further exploited by identification of transgressive segregants in later generations or even through heterosis breeding too. These results are in accordance with findings of Verma and Srivasthava (2004) and Srijan et al., (2016). In rice, the major yield deciding traits are number of productive tillers per plant, number of grains per panicle and 1000 grain weight. Five crosses in case of number of productive tillers per plant, nine for number of grains per panicle and only four for 1000 grain weight exhibited significant positive $S C A$ effects in the present investigation. Among the 5 crosses with high $S C A$ effects for productive tillers per plant, two viz., RP-Bio5478-270 x RP-Bio-5478 -185 (high x low) and DRR Dhan-40 x SM-686 (low x high) also exhibited better per se performance, whereas with respect to the remaining three crosses viz., MTU 1010 x RP-Bio-5478-166 (low x high), MTU 1010 x RP-Bio-5478-176 (low x medium) and WGL-32100 x Ramappa (low $\mathrm{x}$ medium), the mean performance was poor.

With regard to grains per panicle, high $S C A$ effects followed by high per se performance was registered in case of four crosses viz., MTU 1010 x Ramappa (low x high), MTU 1010 x RP-Bio-5478-176 (low x low), WGL$32100 \times$ Ramappa (high x high) and WGL$32100 \times$ SM-686 (high $\times$ high) where as in rest of the crosses there is no reflection of high mean with high $S C A$ especially in case of WGL-32100 x RP-Bio-5478-176 (high x low) and RP-Bio-5478-166 x DRR Dhan-40 (low x low). 
Table.1 Analysis of variance (mean squares) for combining ability and proportions of $G C A$ and $S C A$ variances for grain yield and its components

\begin{tabular}{|c|c|c|c|c|c|c|c|c|c|c|}
\hline Source of variation & d. f. & $\begin{array}{c}\text { Days to } \\
50 \% \\
\text { flowering }\end{array}$ & $\begin{array}{c}\text { Plant } \\
\text { height }\end{array}$ & $\begin{array}{c}\text { Total no. } \\
\text { of } \\
\text { tillers/plan } \\
\mathbf{t}\end{array}$ & $\begin{array}{c}\text { No. of } \\
\text { productive } \\
\text { tillers/ } \\
\text { plant }\end{array}$ & $\begin{array}{l}\text { Panicle } \\
\text { length }\end{array}$ & $\begin{array}{l}\text { No. of } \\
\text { grains/ } \\
\text { panicle }\end{array}$ & $\begin{array}{l}\text { Spikelet } \\
\text { fertility }\end{array}$ & $\begin{array}{c}1000 \text { grain } \\
\text { weight }\end{array}$ & $\begin{array}{c}\text { Grain yield } \\
\text { plant }\end{array}$ \\
\hline GCA & 9 & $36.974 * *$ & $825.843 * *$ & $13.24 * *$ & $6.18 * *$ & $7.68 * *$ & $2059.80 * *$ & $28.33 * *$ & $16.70 * *$ & $48.59 * *$ \\
\hline SCA & 45 & $19.173 * *$ & $260.980 * *$ & $4.12 * *$ & $3.00 * *$ & $3.04 * *$ & $452.91 * *$ & 6.05 & $6.14 * *$ & $7.20 * *$ \\
\hline Error & 108 & 1.65 & 11.11 & 0.70 & 0.60 & 0.64 & 49.64 & 4.35 & 0.50 & 1.10 \\
\hline$\sigma^{2} \mathrm{GCA}$ & & 2.943 & 67.894 & 1.045 & 0.465 & 0.587 & 167.51 & 1.99 & 1.350 & 3.957 \\
\hline$\sigma^{2}$ sca & & 17.514 & 249.869 & 3.418 & 2.408 & 2.398 & 403.26 & 1.70 & 5.636 & 6.099 \\
\hline$\sigma^{2} \mathrm{GCA} / \sigma^{2} \mathrm{sca}$ & & 0.168 & 0.272 & 0.306 & 0.193 & 0.245 & 0.415 & 1.175 & 0.240 & 0.649 \\
\hline
\end{tabular}

Table. 2 Mean sum of squares yield and yield components of ten parents and forty five $\mathrm{F}_{1} \mathrm{~s}$ in rice (Combining ability analysis)

\begin{tabular}{|c|c|c|c|c|c|c|c|c|c|c|}
\hline Source of variation & d.f. & $\begin{array}{c}\text { Days to } \\
50 \% \\
\text { flowering }\end{array}$ & $\begin{array}{l}\text { Plant } \\
\text { height }\end{array}$ & $\begin{array}{c}\text { Total no. } \\
\text { of } \\
\text { tillers/plan } \\
t\end{array}$ & $\begin{array}{c}\text { No. of } \\
\text { productive } \\
\text { tillers/ plant }\end{array}$ & $\begin{array}{l}\text { Panicle } \\
\text { length }\end{array}$ & $\begin{array}{c}\text { No. of } \\
\text { grains/panicl } \\
\text { e }\end{array}$ & $\begin{array}{l}\text { Spikelet } \\
\text { fertility }\end{array}$ & $\begin{array}{l}1000 \text { grain } \\
\text { weight }\end{array}$ & $\begin{array}{c}\text { Grain yield/ } \\
\text { plant }\end{array}$ \\
\hline Replications & 2 & 1.65 & 29.90 & 0.82 & 0.90 & 2.51 & 162.66 & 29.95 & 1.02 & 2.67 \\
\hline Genotypes & 54 & $66.41 * *$ & $1065.37 * *$ & $16.93 * *$ & $10.61 * *$ & $11.45^{* *}$ & $2162.17 * *$ & $29.31 * *$ & $23.71 * *$ & $42.31 * *$ \\
\hline Parents & 9 & $78.88 * *$ & $878.34 * *$ & $11.25^{* *}$ & $3.61^{*}$ & $16.76^{* *}$ & $3465.35 * *$ & $58.56^{* *}$ & $17.53 * *$ & $59.64 * *$ \\
\hline Hybrids & 44 & $62.30 * *$ & $1127.59 * *$ & $17.70 * *$ & $12.10 * *$ & $9.41 * *$ & $1943.18 * *$ & $23.75 * *$ & $22.20 * *$ & $37.35 * *$ \\
\hline Parent Vs. Hybrids & 1 & $135.33 * *$ & $10.96 *$ & $34.02 * *$ & $7.71 *$ & $53.50 * *$ & $69.32 *$ & $10.83 *$ & $145.43 * *$ & $104.59 * *$ \\
\hline Error & 108 & 4.97 & 33.33 & 2.11 & 1.80 & 2.33 & 148.93 & 13.07 & 21.51 & 3.32 \\
\hline
\end{tabular}

* Significant at $5 \%$ level, ** Significant at $1 \%$ level 
Table.3 General combining ability (gca) effects of parents for yield and yield components in rice

\begin{tabular}{|c|c|c|c|c|c|c|c|c|c|}
\hline Parents & $\begin{array}{c}\text { Days to } 50 \% \\
\text { flowering }\end{array}$ & Plant height & $\begin{array}{l}\text { Total no. of } \\
\text { tillers/plant }\end{array}$ & $\begin{array}{l}\text { No. of productive } \\
\text { tillers/ plant }\end{array}$ & $\begin{array}{l}\text { Panicle } \\
\text { length }\end{array}$ & $\begin{array}{c}\text { No. of } \\
\text { grains/panicle }\end{array}$ & $\begin{array}{l}\text { Spikelet } \\
\text { fertility }\end{array}$ & $\begin{array}{c}1000 \text { grain } \\
\text { weight }\end{array}$ & $\begin{array}{c}\text { Grain yield/ } \\
\text { plant }\end{array}$ \\
\hline MTU 1010 & $1.267 * *$ & $13.717 * *$ & $-1.950 * *$ & $-1.294^{* *}$ & -0.161 & $-5.799 * *$ & $3.864^{* * *}$ & $-1.546 * *$ & $-2.733^{* *}$ \\
\hline WGL-32100 & $-2.900 * *$ & $-9.083 * *$ & $-1.487 * *$ & $-1.006 * *$ & $-0.956 * *$ & $15.851 * *$ & 0.106 & $1.552 * *$ & $2.722 * *$ \\
\hline WGL-23985 & $0.767 *$ & $-6.515 * *$ & -0.008 & 0.189 & -0.084 & $15.322 * *$ & -1.026 & $1.458 * *$ & $2.066^{* *}$ \\
\hline RP-Bio-5478-270 & -0.539 & $-3.656 * *$ & $0.818^{* *}$ & $0.913 * *$ & -0.242 & $11.248 * *$ & -0.627 & $0.666^{* *}$ & $2.105^{* *}$ \\
\hline RP-Bio-5478-166 & $1.794 * *$ & $4.165 * *$ & $1.359^{* *}$ & $0.725^{* *}$ & $-1.108 * *$ & $-10.097 * *$ & -0.657 & $-0.764 * *$ & $-1.481 * *$ \\
\hline RP-Bio-5478-176 & $2.211^{* *}$ & $4.267 * *$ & 0.305 & 0.171 & $-0.483^{*}$ & $-16.022 * *$ & -0.388 & $-1.488 * *$ & $-2.111^{* *}$ \\
\hline DRR Dhan - 40 & $1.489^{* *}$ & $11.670^{* *}$ & -0.177 & 0.031 & 0.801 & $-16.124 * *$ & $-1.348^{*}$ & $-1.130 * *$ & $-1.355 * *$ \\
\hline RP-Bio-5478 -185 & $-0.900 *$ & 0.597 & -0.314 & $-0.460 *$ & $1.317^{* *}$ & $-11.529 * *$ & -1.114 & -0.195 & $-1.383 * *$ \\
\hline SM-686 & $-2.150 * *$ & $-6.016^{* *}$ & $0.977 * *$ & $0.516^{*}$ & $0.961^{* *}$ & $8.810 * *$ & 0.086 & $0.713^{* *}$ & $1.314 * *$ \\
\hline SM-787 & $-1.039 * *$ & $-9.236 * *$ & $0.478^{*}$ & 0.215 & -0.044 & $8.339 * *$ & 1.104 & $0.736^{* *}$ & $0.857^{* *}$ \\
\hline $\mathrm{SE}(\mathrm{gi}) \pm$ & 0.798 & 2.065 & 0.520 & 0.480 & 0.498 & 4.365 & 1.293 & 0.441 & 0.652 \\
\hline
\end{tabular}

Table.4 Specific combining ability (sca) effects of crosses for yield and yield components in rice

\begin{tabular}{|c|c|c|c|c|c|c|c|c|c|}
\hline Cross & $\begin{array}{l}\text { Days to } 50 \% \\
\text { flowering }\end{array}$ & $\begin{array}{c}\text { Plant } \\
\text { height }\end{array}$ & $\begin{array}{l}\text { Total no. of } \\
\text { tillers/plant }\end{array}$ & $\begin{array}{l}\text { No. of productive } \\
\text { tillers/ plant }\end{array}$ & $\begin{array}{l}\text { Panicle } \\
\text { length }\end{array}$ & $\begin{array}{c}\text { No. of } \\
\text { grains/panicle }\end{array}$ & $\begin{array}{l}\text { Spikelet } \\
\text { fertility }\end{array}$ & $\begin{array}{c}1000 \text { grain } \\
\text { weight }\end{array}$ & $\begin{array}{c}\text { Grain } \\
\text { yield/ plant }\end{array}$ \\
\hline MTU-1010 x WGL-32100 & $10.045^{* *}$ & $-14.587 * *$ & -0.788 & -1.028 & -0.233 & $38.847 * *$ & $6.973^{* *}$ & $-5.293 * *$ & -0.750 \\
\hline MTU-1010 x Ramappa & -0.621 & $-16.205^{* *}$ & -0.607 & 0.111 & -0.352 & $21.870 * *$ & $4.636^{*}$ & $-2.949 * *$ & 1.046 \\
\hline MTU-1010 x RP-Bio-5478-270 & $4.018^{* *}$ & $25.458 * *$ & 0.100 & 0.387 & -1.214 & $-25.639 * *$ & -1.420 & $-4.157 * *$ & $-6.912 * *$ \\
\hline MTU-1010 x RP-Bio-5478-166 & $8.018^{* *}$ & $25.729 * *$ & $2.493^{* *}$ & $2.375^{* *}$ & 0.879 & -6.801 & $-4.557^{*}$ & $-2.871 * *$ & $-2.683 * *$ \\
\hline MTU-1010 x RP-Bio-5478-176 & $2.934^{*}$ & $25.333 * *$ & $2.081^{*}$ & $2.295^{* *}$ & 1.480 & $23.260 * *$ & -2.146 & $-1.663^{*}$ & 0.237 \\
\hline MTU-1010 x DRR Dhan - 40 & $6.990 * *$ & $13.560 * *$ & 0.269 & -0.291 & 0.753 & $-17.694 * *$ & -3.562 & -0.361 & $-2.682 * *$ \\
\hline MTU-1010 x RP-Bio-5478 -185 & $-3.955^{* *}$ & -3.034 & $-3.367 * *$ & $-2.907 * *$ & 0.274 & -13.032 & $-5.953 * *$ & $-1.899 * *$ & -1.651 \\
\hline MTU-1010 x SM-686 & $-3.038 *$ & -3.924 & $-1.892 *$ & $-1.549 *$ & -0.587 & -3.862 & 0.443 & $1.942 * *$ & -0.818 \\
\hline MTU-1010 x SM-787 & 1.184 & -3.044 & $-1.892^{*}$ & $-1.915^{*}$ & $-2.805^{* *}$ & 12.037 & -0.204 & 0.743 & -1.651 \\
\hline WGL-32100 x Ramappa & $-5.788 * *$ & -3.555 & $3.344^{* *}$ & $2.956^{* *}$ & 0.213 & $33.537 * *$ & -1.417 & $2.006 * *$ & $2.748^{* *}$ \\
\hline WGL-32100 x RP-Bio-5478-270 & -1.482 & $-6.779^{*}$ & 0.458 & -0.155 & -0.222 & $-52.533 * *$ & -0.619 & $-2.882 * *$ & $-4.765 * *$ \\
\hline WGL-32100 x RP-Bio-5478-166 & -0.816 & $9.765^{* *}$ & $-1.986^{*}$ & -1.047 & 0.414 & $-13.648^{*}$ & -0.533 & $1.971 * *$ & -0.895 \\
\hline WGL-32100 x RP-Bio-5478-176 & -1.899 & -0.390 & $-2.382 * *$ & $-3.494 * *$ & -0.811 & $33.487 * *$ & -0.548 & 0.509 & 0.241 \\
\hline WGL-32100 x DRR Dhan - 40 & $-2.843 *$ & $-15.983 * *$ & 0.606 & $1.603^{*}$ & -1.148 & 0.609 & -1.991 & $2.604 * *$ & $2.432 *$ \\
\hline WGL-32100 x RP-Bio-5478 -185 & $-3.788 * *$ & $21.083 * *$ & $-2.013 *$ & $-1.445^{*}$ & $-1.757 *$ & -3.132 & 0.751 & -0.227 & $2.706^{* *}$ \\
\hline
\end{tabular}




\begin{tabular}{|c|c|c|c|c|c|c|c|c|c|}
\hline \multirow[b]{2}{*}{ Cross } & \multirow[b]{2}{*}{$\begin{array}{c}\text { Days to } 50 \% \\
\text { flowering }\end{array}$} & \multirow[b]{2}{*}{ Plant height } & \multirow[b]{2}{*}{$\begin{array}{l}\text { Total no. of } \\
\text { tillers/plant }\end{array}$} & \multirow[b]{2}{*}{$\begin{array}{l}\text { No. of productive } \\
\text { tillers/ plant }\end{array}$} & \multirow[b]{2}{*}{$\begin{array}{l}\text { Panicle } \\
\text { length }\end{array}$} & \multirow[b]{2}{*}{$\begin{array}{c}\text { No. of grains/ } \\
\text { panicle }\end{array}$} & \multirow[b]{2}{*}{$\begin{array}{l}\text { Spikelet } \\
\text { fertility }\end{array}$} & \multicolumn{2}{|c|}{ (c) } \\
\hline & & & & & & & & $\begin{array}{l}1000 \text { grain } \\
\text { weight }\end{array}$ & $\begin{array}{c}\text { Grain yield/ } \\
\text { plant }\end{array}$ \\
\hline WGL-32100 x SM- 686 & 0.129 & 2.089 & -0.454 & 0.196 & $1.928 *$ & $28.815^{* *}$ & 0.388 & 0.888 & $3.389 * *$ \\
\hline WGL-32100 xSM- 787 & 2.018 & -3.641 & 0.495 & 0.380 & -1.114 & $-21.043 * *$ & -2.020 & -0.419 & -1.364 \\
\hline Ramappa x RP-Bio-5478-270 & $2.518^{*}$ & 2.809 & $-3.688 * *$ & $-2.930 * *$ & 1.482 & $-25.453 * *$ & -1.200 & $-1.408^{*}$ & $-4.509 * *$ \\
\hline Ramappa x RP-Bio-5478-166 & $2.851^{*}$ & $9.073 * *$ & $-2.855 * *$ & $-2.005 * *$ & $3.562 * *$ & 2.275 & 2.883 & $2.145 * *$ & $2.114^{*}$ \\
\hline Ramappa x RP-Bio-5478-176 & $2.434^{*}$ & $-12.512 * *$ & $2.473 * *$ & $2.479 * *$ & $-2.533 * *$ & $-42.067 * *$ & -3.336 & 0.300 & -1.212 \\
\hline Ramappa x DRR Dhan - 40 & $4.157 * *$ & $23.108 * *$ & $-2.546 * *$ & $-2.714 * *$ & 0.643 & -5.498 & 1.128 & 0.515 & -1.758 \\
\hline Ramappa x SM-686 & $2.795^{*}$ & 0.634 & 0.083 & 0.128 & $-2.780 * *$ & $-19.456 * *$ & 0.880 & -0.295 & $-2.898 * *$ \\
\hline Ramappa x SM-787 & $4.351 * *$ & $12.084 * *$ & 1.133 & 0.932 & -0.209 & -6.361 & -0.428 & $-1.991 * *$ & -0.037 \\
\hline RP-Bio-5478-270 x RP-Bio-5478-166 & 2.157 & $-22.637 * *$ & $-2.241 * *$ & -1.133 & -0.794 & 10.506 & 2.013 & 1.283 & $3.112 * *$ \\
\hline RP-Bio-5478-270 x RP-Bio-5478-176 & -2.260 & -2.652 & 0.980 & $1.711^{*}$ & -0.752 & 0.291 & -1.385 & -0.992 & -0.028 \\
\hline RP-Bio-5478-270 x DRR Dhan - 40 & -2.205 & $-26.949 * *$ & -1.252 & -0.272 & $-2.239 * *$ & 11.366 & 2.168 & $1.693^{*}$ & $2.559 *$ \\
\hline RP-Bio-5478-270 x RP-Bio-5478 -185 & $-3.149 *$ & $25.577 * *$ & $5.381 * *$ & $3.413 * *$ & -0.479 & $45.271 * *$ & 1.224 & $2.318 * *$ & 1.597 \\
\hline RP-Bio-5478-270 x SM-686 & $2.434^{*}$ & -0.056 & -0.223 & 0.077 & 1.201 & $14.435^{*}$ & 0.100 & 0.083 & -0.657 \\
\hline RP-Bio-5478-270 x SM-787 & & & & & & & & \multicolumn{2}{|c|}{ (Cont.) } \\
\hline Cross & $\begin{array}{c}\text { Days to } 50 \% \\
\text { flowering }\end{array}$ & $\begin{array}{l}\text { Plant } \\
\text { height }\end{array}$ & $\begin{array}{l}\text { Total no. of } \\
\text { tillers/plant }\end{array}$ & $\begin{array}{c}\text { No. of } \\
\text { productive } \\
\text { tillers/ plant }\end{array}$ & $\begin{array}{l}\text { Panicle } \\
\text { length }\end{array}$ & $\begin{array}{l}\text { No. of } \\
\text { grains/ } \\
\text { panicle }\end{array}$ & $\begin{array}{l}\text { Spikelet } \\
\text { fertility }\end{array}$ & $\begin{array}{l}1000 \text { grain } \\
\text { weight }\end{array}$ & $\begin{array}{c}\text { Grain yield/ } \\
\text { plant }\end{array}$ \\
\hline RP-Bio-5478-166 x RP-Bio-5478-176 & -1.927 & $-13.702 * *$ & 0.946 & -0.391 & 0.714 & $14.862 *$ & -1.925 & $1.498^{*}$ & 0.738 \\
\hline RP-Bio-5478-166 x DRR Dhan - 40 & $3.129 *$ & $-16.871 * *$ & -0.329 & -0.251 & $-3.220 * *$ & $16.781 *$ & -0.995 & $-2.044 * *$ & -0.034 \\
\hline RP-Bio-5478-166 x RP-Bio-5478 -185 & $-4.482 * *$ & $-8.108 *$ & 0.161 & 0.467 & -0.466 & 1.639 & 2.444 & 1.261 & 1.423 \\
\hline RP-Bio-5478-166 x SM- 686 & $-5.566 * *$ & -4.818 & 0.883 & 0.295 & $-2.983 * *$ & $-20.923 * *$ & 0.387 & $-6.007 * *$ & $-2.581 *$ \\
\hline RP-Bio-5478-166 x SM-787 & $-3.677 * *$ & 0.434 & -1.174 & -0.421 & -0.518 & -0.875 & 1.070 & 0.590 & -1.530 \\
\hline RP-Bio-5478-176 x DRR Dhan - 40 & -0.621 & $31.897 * *$ & 0.998 & 0.303 & $2.692 * *$ & -5.888 & 2.436 & $-2.502 * *$ & 0.799 \\
\hline RP-Bio-5478-176 x RP-Bio-5478 -185 & -1.566 & $26.376 * *$ & $-4.445 * *$ & $-3.262 * *$ & 0.009 & -6.216 & 1.935 & -0.134 & 0.756 \\
\hline RP-Bio-5478-176 x SM-686 & 0.018 & $-8.091 *$ & $-1.779 *$ & -0.962 & -1.362 & $-23.305 * *$ & 0.628 & -0.402 & $-2.611 * *$ \\
\hline RP-Bio-5478-176 x SM-787 & $-3.760 * *$ & $-10.731 * *$ & 0.143 & 0.009 & $1.680 *$ & $-19.750 * *$ & -1.509 & $-2.162 * *$ & -0.464 \\
\hline DRR Dhan - 40 x RP-Bio-5478 -185 & 1.490 & $-15.330 * *$ & -0.297 & -0.859 & $-2.621 * *$ & 3.253 & 0.949 & 0.838 & -0.773 \\
\hline DRR Dhan - 40 x SM-686 & 0.073 & -5.260 & $1.736^{*}$ & $2.162 * *$ & -0.155 & -0.253 & -1.795 & $-1.487 *$ & 0.690 \\
\hline DRR Dhan - 40 x SM-787 & -2.371 & $-20.518 * *$ & $-2.065^{*}$ & $-2.074 * *$ & -0.301 & 7.085 & 0.871 & -0.697 & -0.419 \\
\hline RP-Bio-5478 -185 x SM-686 & 1.795 & $-8.198 *$ & 0.716 & 0.943 & $1.662 *$ & -9.515 & -1.322 & -0.632 & $-2.566^{*}$ \\
\hline $\mathrm{SE}(\mathrm{Sij}) \pm$ & 2.391 & 6.188 & 1.560 & 1.438 & 1.494 & 13.080 & 3.876 & 1.321 & 1.954 \\
\hline
\end{tabular}

* Significant at $5 \%$ level, ** Significant at $1 \%$ level 
The high SCA observed in these 4 crosses is ascribed to prevalence of non-additive genetic effects and also interaction effects of dominant + and - alleles especially of additive $\mathrm{x}$ dominant or dominant $\mathrm{x}$ additive type. Therefore, these crosses could be better exploited by inter mating in early generations and postponing the selections to later generations (Table 4 and 5).

It is interesting to note that the promising crosses with high test weight (more than $20.00 \mathrm{~g}$ ) also manifested high SCA effects which is an indicative of positive relation between these two estimates in case of 1000 grain weight. The best specific crosses identified for further improvement are WGL32100 x Ramappa (high x high), WGL-32100 $x$ DRR Dhan - 40 (high x low), Ramappa $x$ RP-Bio-5478-166 (high x low), RP-Bio-5478$270 \times$ RP-Bio-5478 -185 (high x low) and among these four, improvement for test weight could be quite easy and straight forward in case of a particular cross (WGL$32100 \times$ Ramappa), in which the parents with high x high GCA effects were involved.

Only two crosses viz., MTU 1010 x WGL32100 (high x medium) and MTU 1010 x Ramappa (high $\mathrm{x}$ low) have shown significant $S C A$ effects for spikelet fertility.

Grain yield is the ultimate result, which is dependent on its components. Graffius (1959) suggested that there would be no separate gene system for yield per se and yield is an end product of multiplicative interaction between yield and its components. In the present study, significant $S C A$ effects were registered by 8 crosses for grain yield per plant and the mean values were also high for 5 crosses. The top ranking cross WGL-32100 x Ramappa which expressed high $S C A$ for grain yield also exhibited high $S C A$ effects for other prime yield components viz., 1000 grain weight, number of grains per panicle and number of productive tillers per plant, whereas, in case of other crosses high SCA effect was observed for at least one component.

In 3 promising crosses viz., WGL-32100 $\mathrm{x}$ Ramappa, WGL-32100 x SM-686, RP-Bio5478-270 x SM-787, the corresponding GCA effects of the parents were of high $\mathrm{x}$ high nature. In rest of the crosses, the parents possessed either high $\mathrm{x}$ low or low $\mathrm{x}$ high $G C A$ effects. The present study indicated that high mean performance is a good indicator of high $G C A$ effects but not an indicator for high $S C A$. Hence, the SCA effects alone may not be an appropriate choice for deciding the breeding strategy wherein considerable importance has to be given for corresponding $G C A$ of the parents apart from mean values of the parents and respective crosses too.

Accordingly, the crosses with good $\mathrm{x}$ good general combiners exhibiting high SCA effects can be utilized for improvement through single plant selection in segregating generations. In other crosses, having high $S C A$ effects due to good $\mathrm{x}$ poor general combiners, the improvement would be possible through population improvement duly involving both additive and dominant gene actions. In case of the crosses showing high SCA effects with involvement of poor $\mathrm{x}$ poor general combiners, the heterosis breeding would be more appropriate.

Thinking in these lines, 3 crosses viz., WGL32100 x Ramappa, WGL-32100 x SM-686, RP-Bio-5478-270 x SM-787 which majorly involved additive gene action are recommended for grain yield improvement through direct selection. Whereas, for other promising crosses, WGL-32100 x DRR Dhan-40, Ramappa x RP-Bio-5478-166, RPBio-5478-270 x RP-Bio-5478-185 (for 1000 grain weight), MTU 1010 x RP-Bio-5478-176 (for number of grains per panicle) and RP- 
Bio-5478-270 x RP-Bio-5478-185, DRR Dhan-40 x SM-686, MTU 1010 x RP-Bio5478-166 (for number of productive tillers per plant), MTU 1010 x RP-Bio-5478-185, RPBio-5478-270 x DRR Dhan-40, RP-Bio-5478176 x SM-787, DRR Dhan-40 x RP-Bio5478-185 (for earliness), population improvement schemes like Bi parental mating and Recurrent selections to pool up the dominant desirable genes by breaking the linkages, if any, would be highly desirable. In future advanced generations such high $\mathrm{x}$ low combinations offer opportunity to practice selection on the transgressive segregants in the desired direction (Langham, 1961).

\section{References}

Arunachalam V 1976. Evaluation of diallel crosses by graphical and combining ability methods, Indian Journal of
Genetics and Plant Breeding 36: 358366.

Graffius, J.E. 1959. Heterosis in barley. Agronomy Journal. 51: 551-554.

Griffing B 1956b. A generilsed treatment of the use of diallel crosses in quantitative inheritance. Heredity 10: 31-50.

Langham, D. G. 1961. Crop Science. 1/3763678.

Srijan, A., Sudheer Kumar, S and Damodar Raju, Ch. 2016. Studies on Gene Action and Combining Ablility in Rice (Oryza sativa L.) Environment \& Ecology. 34 (4B): 1749-1755.

Verma, O. P and Srivasthava, H.K. 2004. Genetic component and combining ability analysis in relation to heterosis for yield and associated traits using three diverse rice growing ecosystems. Field Crops Research. 88: 91-102.

\section{How to cite this article:}

Lingaiah N., N. Sarla, V. Venkanna, Ch. Surender Raju, K. Radhika and Vishnu Vardhan Reddy D. 2018. Combining Ability Analysis in Iron and Zinc Rich Rice Genotypes. Int.J.Curr.Microbiol.App.Sci. 7(05): 3745-3751. doi: https://doi.org/10.20546/ijcmas.2018.705.434 